\title{
ON KNESER'S ADDITION THEOREM IN GROUPS
}

\author{
GEORGE T. DIDERRICH
}

Abstract. The following theorem is proved.

THEOREM A. Let $G$ be a group written additively with finite nonempty subsets $A, B$. Assume that $B$ is commutative, i.e. $b_{1}+b_{2}=$ $b_{2}+b_{1}$, for $b_{1}, b_{2} \in B$. Then there exists an Abelian subgroup $H$ of $G$ such that $A+B+H=A+H+B=A+B$ and $|A+B| \geqq|A+H|+$ $|B+H|-|H|$.

This is Kneser's theorem, if $G$ is Abelian. Also, as an application of the above theorem, the following is proved.

THEOREM B. Let $G$ be a finite group of order $v(v>1)$ and let $a_{1}, \cdots, a_{v}$ be a sequence (repeats are allowed) of nonzero elements of $G$. The set $S$ of sums $a_{i_{1}}+\cdots+a_{i_{t}}$ where $1 \leqq i_{1}<\cdots<i_{t} \leqq v$ and $1 \leqq t \leqq v$ must contain a nontrivial subgroup $H$ of $G$.

Finally, the Kemperman $d$-transform, a transform similar to the Dyson $e$-transform, is introduced and evidence is given to support the conjecture that Theorem A remains true, if the commutativity of $B$ is dropped.

1. Introduction. Let $G$ be an arbitrary group written additively, and let $A, B$, and $C$ be finite nonempty subsets of $G . A+B$, the Schnirelmann sum of $A$ and $B$, denotes the set of sums of the form $a+b$ where $a \in A$ and $b \in B$. A set $B$ in $G$ is said to be commutative, if $b_{2}+b_{1}=b_{1}+b_{2}$ for $b_{1}, b_{2} \in B$. Also, $|A|$ denotes the cardinal number of elements in $A$. Finally, for a set $B$ the notation $\langle B\rangle$ denotes the group generated by $B$. We follow the notation and terminology in Mann [3] and Kemperman [1] and [2].

In $\$ 2$ we prove that Kneser's theorem (cf. Theorem 1.5 in [3]) holds in an arbitrary group provided that one of the component sets is commutative. We prove

TheOREM 1. Let $G$ be an arbitrary group and let $A, B$ denote finite nonempty subsets of $G$. Assume that $B$ is commutative. Then there exists an Abelian subgroup $H$ of $G$ such that

$$
\begin{gathered}
A+B+H=A+H+B=A+B ; \\
|A+B| \geqq|A+H|+|B+H|-|H| .
\end{gathered}
$$

Received by the editors September 7, 1972 and, in revised form, September 26, 1972. AMS (MOS) subject classifications (1970). Primary 20N99; Secondary $10 \mathrm{~J} 99$.

Key words and phrases. Addition theorems, Kneser theorem, the Dyson e-transform, the Kemperman $d$-transform, commutative sets. 
Theorem 1 is Kneser's theorem, if $G$ is Abelian. In $\S 3$ we give an application of Theorem 1, and in $\S 4$ we discuss the conjecture that Theorem 1 is true, if the commutativity of $B$ is omitted.

We now introduce the Dyson $e$-transform (cf. pp. 5-6 in [3]) and show that its main properties hold, if $B$ is commutative.

Definition 1.1. Let $G$ be a group with finite nonempty subsets $A$ and $B$. Assume that $B$ is commutative and $0 \in B$. Fix $e \in A$ and define the sets

$$
\begin{aligned}
& A^{\prime}=A \cup(e+B), \\
& B^{\prime}=B \cap(-e+A) .
\end{aligned}
$$

We call $A^{\prime}, B^{\prime}$ the Dyson e-transform of $A, B$ respectively.

Lemma 1.2. Let $A^{\prime}, B^{\prime}$ be the Dyson e-transform of $A, B$. Then

(a) $A^{\prime} \supseteq A$ and $B^{\prime} \subseteq B$,

(b) $A^{\prime}+B^{\prime} \subseteq A+B$,

(c) $\left|A^{\prime}\right|+\left|B^{\prime}\right|=|A|+|B|$.

Proof. Part (a) of the lemma clearly holds. For (c), it suffices to prove that $x \in A^{\prime} \backslash A$ iff $-e+x \in B \backslash B^{\prime}$. If $x \in A^{\prime} \backslash A$, then for some $b \in B$, we have $x=e+b$; hence $-e+x=b$ and $b \notin B^{\prime}$, otherwise for some $a \in A$ we would have $-e+x=-e+a$, thus $x=a$, a contradiction. So, $x \in A^{\prime} \backslash A$ implies that $-e+x \in B \backslash B^{\prime}$. Conversely, if $-e+x \in B \backslash B^{\prime}$, then for some $b \in B$ we have $-e+x=b$, so $x=e+b$ and $x \notin A$, otherwise for some $a \in A$ we would have $x=a=e+b$, hence $-e+x \in B^{\prime}$, a contradiction. Thus we have proved $x \in A^{\prime} \mid A$ iff $-e+x \in B \mid B^{\prime}$, so $\left|A^{\prime}\right| A \mid=$ $\left|B \backslash B^{\prime}\right|$ which proves (c).

For (b), if $x \in A$, then $x+B^{\prime} \subseteq A+B$. So we may assume that $x \in A^{\prime} \backslash A$, hence $x=e+b$ for some $b \in B$. Now $x+B^{\prime}=e+b+B^{\prime}$, but $b$ and $B^{\prime}$ commute because $B^{\prime} \subseteq B$, so $x+B^{\prime}=\left(e+B^{\prime}\right)+b$. However, $B^{\prime} \subseteq-e+A$, so $e+B^{\prime} \subseteq A$. Consequently, $x+B=\left(e+B^{\prime}\right)+b \subseteq A+b \subseteq A+B$ which proves (b). This completes the proof of the lemma.

Since the Dyson $e$-transform holds, if $B$ is commutative, we proceed in a straightforward way (with some minor modifications) following Mann's proof (cf. pp. 6-9 in [3]). Further, we introduce some additional notation. For a set $A$ in $G, \bar{A}$ denotes the complement of $A$ in $G$, i.e. $\bar{A}=G \backslash A$; and for a subgroup $H$ of $G, N(H)$ denotes the normalizer of $H$ in $G$, i.e. $N(H)=\{x \in G \mid x+H=H+x\}$.

2. The lemmas and proof of Theorem 1. Lemmas 2.0, 2.1, and 2.2 below are analogous to Lemmas 1.5.1, 1.5.2, and 1.5.3 respectively in Mann [3]. The main point is that the properties of the Dyson $e$-transform hold, if $B$ is commutative. Hence, by repeatedly applying the $e$-transform, we 
can "essentially" reduce the problem to the case when $A+B=A$. The choice of $H=\langle B\rangle$ then clearly satisfies (1) and (2) in the theorem.

LEMMA 2.0. Let $G$ be a group with subgroups $H_{1}$ and $H_{2}$, and let $C_{1}$ and $C_{2}$ be finite nonempty subsets of $G$ such that

(a) $C_{1}+H_{1}=C_{1}$,

(b) $\mathrm{C}_{2}+\mathrm{H}_{2}=\mathrm{C}_{2}$,

(c) $N\left(H_{1}\right) \supseteq H_{2}$ and $N\left(H_{2}\right) \supseteq H_{1}$, and

(d) neither $\bar{C}_{1} \cap C_{2}$ nor $\bar{C}_{2} \cap C_{1}$ is empty.

Then either

$$
\begin{aligned}
& \left|\bar{C}_{1} \cap C_{2}\right| \geqq\left|H_{1}\right|-\left|H_{1} \cap H_{2}\right| \text { or } \\
& \left|\bar{C}_{2} \cap C_{1}\right| \geqq\left|H_{2}\right|-\left|H_{1} \cap H_{2}\right| .
\end{aligned}
$$

Proof. We first observe that $H_{1}+H_{2}$ is a group with finite normal subgroups $H_{1}, H_{2}$ and $H_{1}+H_{2}=H_{2}+H_{1}$. This follows from (c) in the lemma. Put $h_{1}=\left|H_{1}\right|$ and $h_{2}=\left|H_{2}\right|$. Let $H^{*}=H_{1}+H_{2}$ and $H=H_{1} \cap H_{2}$ and put $h=|H|$. Since $H^{*} / H_{2} \cong H_{1} / H$ and $H^{*} / H_{1} \cong H_{2} / H$, we have $m_{2} h=h_{1}$ and $m_{1} h=h_{2}$ where $m_{2}=\left|H^{*}\right| H_{2} \mid$ and $m_{1}=\left|H^{*}\right| H_{1} \mid$. The relations (1) and (2) become

$$
\begin{aligned}
& \left|\bar{C}_{1} \cap C_{2}\right| \geqq h_{1}-h=h\left(m_{2}-1\right) ; \\
& \left|\bar{C}_{2} \cap C_{1}\right| \geqq h_{2}-h=h\left(m_{1}-1\right) .
\end{aligned}
$$

Without loss of generality we may assume that $m_{2} \geqq m_{1}$. Let $e \in \bar{C}_{2} \cap C_{1}$ and form the $H^{*}$-coset $R=e+H^{*} . R$ is a union of $m_{1}$ disjoint $H_{1}$-cosets as well as a union of $m_{2} H_{2}$-cosets. Conditions (a) and (b) in the lemma imply that $C_{1}$ is a union of $H_{1}$-cosets and $C_{2}$ is a union of $H_{2}$-cosets. Assume that $C_{1} \cap R$ consists of $v_{1}$ disjoint $H_{1}$-cosets and $C_{2} \cap R$ consists of $v_{2}$ disjoint $H_{2}$-cosets. Then $\bar{C}_{1} \cap R$ consists of $m_{1}-v_{1}$ disjoint $H_{1}$-cosets and $\bar{C}_{2} \cap R$ consists of $m_{2}-v_{2}$ disjoint $H_{2}$-cosets. However, one readily verifies using (c) in the lemma that the intersection of an $H_{1}$-coset and an $H_{2}$-coset in $R$ is exactly an $H$-coset. Thus $\left(\bar{C}_{1} \cap R\right) \cap\left(C_{2} \cap R\right)$ is a union of $\left(m_{1}-v_{1}\right) v_{2}$ disjoint $H$-cosets and $\left(\bar{C}_{2} \cap R\right) \cap\left(C_{1} \cap R\right)$ is a union of $\left(m_{2}-v_{2}\right) v_{1}$ disjoint $H$-cosets. This yields

$$
\begin{aligned}
& \left|\bar{C}_{1} \cap C_{2} \cap R\right|=\left(m_{1}-v_{1}\right) v_{2} h ; \\
& \left|\bar{C}_{2} \cap C_{1} \cap R\right|=\left(m_{2}-v_{2}\right) v_{1} h>0 .
\end{aligned}
$$

The inequality in (6) is justified by choice of $e$. 
From (3), (4), (5), and (6) it suffices to prove either

$$
\begin{aligned}
& \left(m_{1}-v_{1}\right) v_{2} \geqq m_{2}-1 \quad \text { or } \\
& \left(m_{2}-v_{2}\right) v_{1} \geqq m_{1}-1 .
\end{aligned}
$$

From (6) we have $v_{1} \geqq 1$ and $m_{2} \geqq v_{2}+1$.

Now we must have $v_{2} \geqq 1$, for if $v_{2}=0$ then (8) holds because $m_{2} \geqq m_{1}$. Also, we must have $m_{1} \geqq v_{1}+1$, for if $m_{1}=v_{1}$ then again (8) holds. Consequently from the above we have

$$
v_{1} \geqq 1, \quad v_{2} \geqq 1, \quad m_{1} \geqq v_{1}+1, \quad \text { and } \quad m_{2} \geqq v_{2}+1 .
$$

If we deny (7) and (8) and add, we get using (9)

$$
\begin{aligned}
2 v_{1} v_{2}-2 & >m_{1} v_{2}+m_{2} v_{1}-m_{1}-m_{2}=m_{1}\left(v_{2}-1\right)+m_{2}\left(v_{1}-1\right) \\
& \geqq\left(v_{1}+1\right)\left(v_{2}-1\right)+\left(v_{2}+1\right)\left(v_{1}-1\right)=2 v_{1} v_{2}-2,
\end{aligned}
$$

which is a contradiction. This proves the lemma.

Lemma 2.1. Let $G$ be a group with finite nonempty subsets $A$ and $B$ Assume that $B$ is commutative. Let $c_{0}$ be a fixed element of $A+B$ where $c_{0}=a_{0}+b_{0}, a_{0} \in A$ and $b_{0} \in B$. Then there exist sets $A_{1}, B_{1}$, and an Abelian subgroup $H_{1}$ of $G$ satisfying

(a) $c_{0} \in A_{1}+B_{1} \subseteq A+B$,

(b) $A_{1} \supseteq A$ and $B_{1} \subseteq B$ (hence $B_{1}$ is commutative),

(c) $\left|A_{1}\right|+\left|B_{1}\right|=|A|+|B|$,

(d) $H_{1}+B_{1}=B_{1}+H_{1}$ and $H_{1}$ is a subgroup of $\langle B\rangle$,

(e) $A_{1}+B_{1}+H_{1}=A_{1}+H_{1}+B_{1}=A_{1}+B_{1}$, and

(f) $\left|A_{1}+B_{1}\right| \geqq\left|A_{1}\right|+\left|B_{1}\right|-\left|H_{1}\right|$.

Hence from (c) and (f), $\left|A_{1}+B_{1}\right| \geqq|A|+|B|-\left|H_{1}\right|$.

Proof. Without loss of generality, we may assume that $a_{0}=b_{0}=c_{0}=0$. For let $A^{\prime}=-a_{0}+A$ and let $B^{\prime}=B-b_{0}$. Note that $B^{\prime}$ is commutative because $B$ is. Suppose we are supplied by the conclusion of this lemma (for $A^{\prime}, B^{\prime}$ in place of $A, B$ respectively) sets $A_{1}^{\prime}, B_{1}^{\prime}$, and an Abelian subgroup $H_{1}^{\prime}$ satisfying (a) through (f). Then one easily verifies that the choice $A_{1}=a_{0}+A_{1}^{\prime}, B_{1}=B_{1}^{\prime}+b_{0}$, and $H_{1}=H_{1}^{\prime}$ satisfies (a) through (f).

If $A+B=A$, then the choice $A_{1}=A, B_{1}=B$, and $H_{1}=\langle B\rangle$ satisfies (a) through (f). Hence, we may assume that $A+B \nsubseteq A$. Consequently, there exists $e \in A$ such that $e+B \nsubseteq A$. Fix such an $e$ and consider the Dyson $e$-transform. From Lemma 1.2 we have

$$
\begin{aligned}
& A^{\prime} \supset A \text { and } B^{\prime} \subset B, \quad\left|A^{\prime}\right|+\left|B^{\prime}\right|=|A|+|B|, \\
& 0 \in A^{\prime}+B^{\prime} \subseteq A+B, \quad\left|B^{\prime}\right|<|B|,
\end{aligned}
$$


where $A^{\prime}, B^{\prime}$ is the Dyson e-transform of $A, B$. Then by induction on $|B|$ we are done. This proves the lemma.

One sees that, for each $c \in A+B$, Lemma 2.1 yields an "inner approximation" to $C=A+B$ satisfying (1) and (2) in the theorem. The next lemma combines both Lemma 2.0 and Lemma 2.1 to piece all of these inner approximations together.

LeMma 2.2. Let $G$ be a group with finite nonempty subsets $A$ and $B$. Assume that $B$ is commutative. For each subset $\left\{c_{1}, \cdots, c_{k}\right\} \subseteq A+B$, there exists a set $C_{0}$ and an Abelian subgroup $H_{0}$ satisfying

(a) $\mathrm{C}_{0}+\mathrm{H}_{0}=\mathrm{C}_{0}$,

(b) $H_{0}+B=B+H_{0}$ and $H_{0}$ is a subgroup of $\langle B\rangle$,

(c) $\left\{c_{1}, \cdots, c_{k}\right\} \subseteq C_{0} \subseteq A+B$, and

(d) $\left|C_{0}\right| \geqq|A|+|B|-\left|H_{0}\right|$.

Proof. We use induction on $k$. For $k=1$ the lemma follows from Lemma 2.1. For $k>1$, let $C_{1}, H_{1}$ satisfy (a) through (d) in place of $C_{0}, H_{0}$ where $\left\{c_{1}, \cdots, c_{k-1}\right\} \subseteq C_{1}$, and similarly let $C_{2}, H_{2}$ satisfy (a) through (d) where $c_{k} \in C_{2}$. If either $C_{1} \subseteq C_{2}$ or $C_{2} \subseteq C_{1}$ we are done. Hence, we may assume that neither $\bar{C}_{1} \cap C_{2}$ nor $\bar{C}_{2} \cap C_{1}$ is empty. Since $H_{1}$ and $H_{2}$ are Abelian subgroups in $\langle B\rangle$, we may apply Lemma 2.0. Thus, either

$$
\begin{aligned}
& \left|\bar{C}_{1} \cap C_{2}\right| \geqq\left|H_{1}\right|-\left|H_{1} \cap H_{2}\right| \text { or } \\
& \left|\bar{C}_{2} \cap C_{1}\right| \geqq\left|H_{2}\right|-\left|H_{1} \cap H_{2}\right| .
\end{aligned}
$$

If (12) holds, let $H_{0}=H_{1} \cap H_{2}$ and $C_{0}=C_{1} \cup C_{2}$. Then $C_{0}+H_{0}=C_{0}$ and $\left\{c_{1}, \cdots, c_{k}\right\} \subseteq C_{0} \subseteq A+B$; furthermore $C_{0}=C_{1} \cup\left(\bar{C}_{1} \cap C_{2}\right)$ so $\left|C_{0}\right|=\left|C_{1}\right|+$ $\left|\bar{C}_{1} \cap C_{2}\right|$. But $\left|C_{1}\right| \geqq|A|+|B|-\left|H_{1}\right|$ and from (12) we get $\left|C_{0}\right| \geqq|A|+$ $|B|=\left|H_{0}\right|$. If (13) holds the proof is similar. This completes the proof of the lemma.

To prove the theorem, take $\left\{c_{1}, \cdots, c_{k}\right\}=A+B$ in Lemma 2.2. Inequality (2) in the theorem follows by considering $A+H, B+H$ in place of $A, B$ respectively, since $(A+H)+(H+B)=A+B$.

3. An application of Theorem 1. Examples of commutative sets $B$ in a group $G$ are subsets of the cyclic group $\langle d\rangle$ where $d \in G$ and $d \neq 0$. In particular sets in the form $\{0, d, \cdots, s d\}$, i.e. arithmetic progressions with first term 0 , are commutative sets.

As an easy application of Theorem 1, we now prove

THEOREM 3. Let $G$ be a finite group of order $v(v>1)$ and let $a_{1}, \cdots, a_{v}$ be a sequence (repeats are allowed) of nonzero elements of $G$. The set $S$ of sums $a_{i_{1}}+\cdots+a_{i_{t}}$ where $1 \leqq i_{1}<\cdots<i_{t} \leqq v$ and $1 \leqq t \leqq v$ must include a nontrivial subgroup $H$ of $G$. 
Proof. Let $S^{\prime}=\left\{0, a_{1}\right\}+\cdots+\left\{0, a_{v}\right\}$. Then $S^{\prime}=S$ unless $0 \notin S$. But $0 \in S$ by the following pyramidal argument. Write

$$
\begin{aligned}
& s_{1}=a_{1} \\
& s_{2}=a_{1}+a_{2} \\
& \cdot \\
& \cdot \\
& \cdot \\
& s_{v}=a_{1}+\cdots+a_{v} .
\end{aligned}
$$

If all the $s_{i}$ 's are distinct then $S=G$, hence $0 \in S$. And, if two are equal say $s_{i}=s_{j}(i<j)$, then $a_{i+1}+\cdots+a_{j}=0$ and again $0 \in S$. Thus $S^{\prime}=S$ and to prove the theorem we need only estimate $S^{\prime}$ by Theorem 1 , since each $\left\{0, a_{i}\right\}$ is commutative.

Put $A_{i}=\left\{0, a_{i}\right\}$ for $1 \leqq i \leqq v$. Theorem 1 implies that for each $2 \leqq j \leqq v$ there is a subgroup $H$ of $G$ such that

$$
\left(A_{1}+\cdots+A_{j}\right)+H=A_{1}+\cdots+A_{j} .
$$

If one such subgroup $H$ were nontrivial, then clearly $S^{\prime} \supset H$ and we are done. If all such subgroups were trivial, then by induction and Theorem 1 we have

$$
\begin{aligned}
\left|A_{1}+\cdots+A_{v}\right| & \geqq\left|A_{1}\right|+\cdots+\left|A_{v}\right|-(v-1) \\
& \geqq 2 v-(v-1)=v+1,
\end{aligned}
$$

a contradiction since $A_{1}+\cdots+A_{v} \subseteq G$ and this concludes the proof of the theorem.

4. The Kemperman $d$-transform and a conjecture. Kemperman (cf. Theorem 5 in [1]) proved the following

Theorem 4. Let $A$ and $B$ be finite nonempty subsets of a semigroup $G$ such that $k(A, B) \geqq 2$ where $k(A, B)=|A|+|B|-|A+B|$. Let $c_{0}=a_{0}+b_{0}$ $\left(a_{0} \in A\right.$ and $\left.b_{0} \in B\right)$ be an element of $A+B$ possessing an inverse in $G$. Then $G$ contains a finite group $H$ such that

$$
\begin{gathered}
|A+B| \geqq|A|+|B|-|H|, \\
a_{0}+H+b_{0} \subseteq A+B .
\end{gathered}
$$

This result is proved by means of a transform similar to the Dyson $e$-transform. We now proceed to define this transform and examine its properties.

Definition 4.1. Let $A, B$ be finite nonempty subsets of an arbitrary 
group $G$. Put $C=A+B$ and $\lambda=|C|-|A|, \mu=|C|-|B|$. Assume that $0 \in A \cap B$. Let $D=A \cap B$ and let $k(A, B)=|A|+|B|-|C|$. Assume that $A+d \notin A$ for some $d \in D$ and fix such a $d$.

Define the sets

$$
\begin{aligned}
& A_{0}=\left\{a_{0} \in A \mid a_{0}+d \notin A\right\}, \\
& B_{0}=\left\{b_{0} \in B \mid d+b_{0} \notin B\right\} .
\end{aligned}
$$

Put $p=\left|A_{0}\right|$ and $q=\left|B_{0}\right|$. From (3) we have $d \neq 0,0 \notin A_{0}, 0 \notin B_{0}$, and $p \geqq 1$. Define the sets $A^{\prime}, B^{\prime}$ by

$$
\begin{array}{ll}
A^{\prime}=A \cup\left(A_{0}+d\right), \quad B^{\prime}=B \backslash B_{0}, & \text { if } p \geqq q ; \\
A^{\prime}=A \backslash A_{0}, \quad B^{\prime}=B \cup\left(d+B_{0}\right), & q>p .
\end{array}
$$

We call $A^{\prime}, B^{\prime}$ the Kemperman d-transform of $A, B$ respectively.

In what follows let $\lambda^{\prime}, \mu^{\prime}$ be defined as $\lambda, \mu$ with $A^{\prime}, B^{\prime}$ in place of $A, B$ respectively. Also, let $C^{\prime}=A^{\prime}+B^{\prime}$.

Lemma 4.2. Let $A^{\prime}, B^{\prime}$ be the Kemperman d-transform of $A, B$. Then

(a) $A^{\prime}+B^{\prime} \subseteq A+B$ and $0 \in A^{\prime} \cap B^{\prime} \subseteq A^{\prime}+B^{\prime}$,

(b) $\left|A^{\prime}\right|+\left|B^{\prime}\right| \geqq|A|+|B|$ with equality iff $p=q$,

(c) $\lambda^{\prime}+\mu^{\prime} \leqq \lambda+\mu$ and either $\lambda^{\prime}<\lambda$ or $\mu^{\prime}<\mu$, and

(d) $k\left(A^{\prime}, B^{\prime}\right) \geqq k(A, B)$.

Proof. For (a), we clearly have $0 \in A^{\prime} \cap B^{\prime} \subseteq A^{\prime}+B^{\prime}$. To show $A^{\prime}+B^{\prime} \subseteq A+B$ first assume that $p \geqq q$ and consider $\left(a_{0}+d\right)+b^{\prime}$ where $a_{0}+d \in A_{0}+d$ and $b^{\prime} \in B^{\prime}$. Then $\left(a_{0}+d\right)+b^{\prime}=a_{0}+\left(d+b^{\prime}\right)$, but $d+b^{\prime} \in B$ by definition of $B_{0}$, so $a_{0}+d+b^{\prime} \in A+B$. The proof for $q>p$ is similar.

For (b), assume that $p \geqq q$. Then $\left|A^{\prime}\right|=|A|+p$ and $\left|B^{\prime}\right|=|B|-q$; hence $\left|A^{\prime}\right|+\left|B^{\prime}\right|=|A|+|B|+(p-q) \geqq|A|+|B|$. The proof for $q>p$ is similar.

For (c), note that $\lambda+\mu=2|C|-|A|-|B|$ and $\lambda^{\prime}+\mu^{\prime}=2\left|C^{\prime}\right|-\left|A^{\prime}\right|-\left|B^{\prime}\right|$, but, by (a), $\left|C^{\prime}\right| \leqq|C|$; hence, by $(b), \lambda^{\prime}+\mu^{\prime} \leqq+\mu$. If $p \geqq q$, then $\left|A^{\prime}\right|>|A|$ hence, by (a), $\left|C^{\prime}\right|-\left|A^{\prime}\right|<|C|-|A|$ thus $\lambda^{\prime}<\lambda$. The proof for $q>p$ is similar.

For (d), we have $k(A, B)=|A|+|B|-|C|$ and $k\left(A^{\prime}, B^{\prime}\right)=\left|A^{\prime}\right|+\left|B^{\prime}\right|-$ $\left|C^{\prime}\right|$. So, by (a) and (b), $k\left(A^{\prime}, B^{\prime}\right) \geqq k(A, B)$.

This completes the proof of the lemma.

Using the Kemperman $d$-transform, we now prove a lemma equivalent to Theorem 4 in the case that $G$ is a group.

Lemma 4.3. Let $G$ be a group with finite nonempty subsets $A$ and $B$. Let $c_{0}$ be a fixed element of $A+B$ where $c_{0}=a_{0}+b_{0}, a_{0} \in A$, and $b_{0} \in B$. 
Then there exist finite subsets $A_{1}, B_{1}$, and a finite subgroup $H_{1}$ of $G$ such that

(a) $A_{1}+B_{1} \subseteq A+B$,

(b) $a_{0} \in A_{1}, b_{0} \in B_{1}$, and $c_{0} \in A_{1}+B_{1}$,

(c) $\left|A_{1}\right|+\left|B_{1}\right| \geqq|A|+|B|$,

(d) $A_{1}+H_{1}+B_{1}=A_{1}+B_{1}$,

(e) either $H_{1} \subseteq\left\langle A_{1}\right\rangle$ or $H_{1} \subseteq\left\langle B_{1}\right\rangle$, and

(f) $\left|A_{1}+B_{1}\right| \geqq\left|A_{1}\right|+\left|B_{1}\right|-\left|H_{1}\right|$.

Hence, from (a), (c), and (f):

$$
|A+B| \geqq|A|+|B|-\left|H_{1}\right| \text {. }
$$

Proof. As in the proof of Lemma 2.1, we may assume that $c_{0}=a_{0}=$ $b_{0}=0$. Thus, $0 \in A \cap B$ and $C=A+B \supseteq A \cup B$, therefore

$$
|C| \geqq|A|+|B|-|D|
$$

where $D=A \cap B$. This implies that $|D| \geqq k(A, B)$. If $k(A, B) \leqq 1$, we are done. So, we may further assume that $|D| \geqq k(A, B) \geqq 2$.

Put $\lambda=|C|-|A|$ and $\mu=|C|-|B|$. Clearly $\lambda \geqq 0$ and $\mu \geqq 0$. If $\lambda=0$, then $A+B=A$. The choice $A_{1}=A, B_{1}=B$, and $H_{1}=\langle B\rangle$ clearly satisfies the conditions in the lemma. Similarly, the lemma holds if $\mu=0$. Further, if $A+D \subseteq A$, then the choice $A_{1}=A, B_{1}=B$, and $H_{1}=\langle D\rangle$ also satisfies the conditions in the lemma where (f) follows from (9). Consequently, we may assume that $A+d \notin A$ for some $d \in D$. Fix such a $d$.

By induction on $\lambda$ and $\mu$, we can assume the lemma holds when $A, B$ is replaced by $A^{\prime}, B^{\prime}$ respectively such that for the corresponding integers $\lambda^{\prime}+\mu^{\prime} \leqq \lambda+\mu$ and either $\lambda^{\prime}<\lambda$ or $\mu^{\prime}<\mu$. By means of the Kemperman $d$-transform (Lemma 4.2), we now construct sets $A^{\prime}, B^{\prime}$ (the Kemperman $d$-transform of $A, B$ ) such that

(1) $A^{\prime}+B^{\prime} \subseteq A+B$ and $0 \in A^{\prime} \cap B^{\prime}$,

(2) $\left|A^{\prime}\right|+\left|B^{\prime}\right| \geqq|A|+|B|$,

(3) $k\left(A^{\prime}, B^{\prime}\right) \geqq k(A, B) \geqq 2$,

(4) $\left|D^{\prime}\right| \geqq 2$, and

(5) $\lambda^{\prime}+\mu^{\prime} \leqq \lambda+\mu$ and either $\lambda^{\prime}<\lambda$ or $\mu^{\prime}<\mu$.

Therefore, by induction this concludes the proof.

Note, Lemma 4.3 is the analogue of Lemma 2.1. Also, Lemma 4.3 implies that

$$
C=\bigcup_{a \in A, b \in B}\left(a+H_{a, b}+b\right)
$$

where for each $a \in A, b \in B, H_{a, b}$ is a subgroup of $G$ satisfying

$$
|C| \geqq|A|+|B|-\left|H_{a, b}\right| \text {. }
$$


Now, if we could prove a lemma analogous to Lemma 2.0 , then we could find a "uniform" $H$ (as in Lemma 2.2) such that

$$
\begin{aligned}
& A+H+B=A+B ; \\
& |C| \geqq|A|+|B|-|H| .
\end{aligned}
$$

So, on the basis of the above results and Theorem 1, we make the Conjecture. Let $G$ be an arbitrary group and let $A, B$ be finite nonempty subsets of $G$. Then $G$ contains a finite group $H$ such that

$$
\begin{gathered}
A+H+B=A+B ; \\
|A+B| \geqq|A+H|+|H+B|-|H| .
\end{gathered}
$$

\section{REFERENCES}

1. J. H. B. Kemperman, On complexes in a semigroup, Nederl. Akad. Wetensch. Proc. Ser. A. 59=Indag. Math. 18 (1956), 247-254. MR 19, 13.

2. - On small sumsets in an abelian group, Acta Math. 103 (1960), 63-88. MR 22 \#1615.

3. H. B. Mann, Addition theorems: The addition theorems of group theory and number theory, Interscience, New York, 1965. MR 31 \#5854.

Current address: 2973 North Cramer Street, Milwaukee, Wisconsin 53211 